SHORT AND LONGER-TERM PLANETARY EFFECTS ON SUN AND EARTH

by

Kenneth W. Dickman; farmer emeritus

\author{
Reprinted from \\ ENERGY \& \\ ENVIRONMENT \\ VOLUME 17 No. 12006
}




\title{
SHORT AND LONGER-TERM PLANETARY EFFECTS ON SUN AND EARTH
}

\author{
Kenneth W. Dickman; farmer emeritus \\ Email: goldco9@tpg.com.au
}

\begin{abstract}
The Solar Electro-magnetic Resonant Cross provides the key to timing of major events on Sun and Earth. Planet/Cross alignments, together with specific interplanetary angular separations, coincided with the seismic events on 24/26 December 2004, of which an outcome was the Asian Tsunami. Planetary conjunctions also coincided with peak sunspot abundance on 30 March 2001, precipitous decay of the giant 1997/8 El Niño in May 1998, and beginning/end (1645/1715) of the last prominent cold period of the Little Ice Age, the Maunder Minimum. Cross and influential angularities promise to be as revealing for the future as the past; because orbits can be calculated in advance. A sharp enhancement of planetary influence on Sun/Earth can be expected in June 2006.
\end{abstract}

\section{INTRODUCTION}

\section{Nothing is really new}

The concept of a solar driver for human affairs is far from new. When heretic pharaoh Akhenaten came to power in $1352 \mathrm{BC}$, he proclaimed the Sun to be the dominant influence on the well-being of Egypt. But the official standing of this eminently plausible hypothesis did not survive the lifetimes of him and Nefertiti. Renewed support for the Sun had to await the observational evidence of variable solar activity enabled by Galileo's telescope. Within 200 years, the obvious correlation between events on Sun and Earth had closed a 3,000-year circle. There is a Sun-Earth connection.

My paper goes a step further, by seeking the underlying driver for this connection. Nothing is entirely new, it seems. Harvard astronomer Owen Gingerich located 276 copies of the 1543 edition of Copernicus' book On the revolutions of the Heavenly Spheres, and was surprised to find that the part about the Earth going around the Sun attracted little attention from peers. Annotations were concentrated on pages relating to the calculation of planetary orbits. Astronomers were seeking an easier way to do this time-consuming task than had been afforded by the Second Century Ptolemaic system of prediction. Planets have long been seen as influential. But how?

\section{Introducing Mercury}

I challenge current theories about the Sun; and offer a reason why it is affected by planetary alignments. Mercury is crucial. It is by far the closest planet, with an orbital 
period of 88 days (next-in-line Venus has a period of 225 days). Furthermore, it has a notably elliptical orbit, matched only by that of distant Pluto. Mercury's orbital eccentricity is 0.206 ( $c f 0.007$ for its stay-at-home neighbour, Venus), and its closest approach to the Sun is when at an azimuth of $75^{\circ}$ - by the conventional $360^{\circ}$ solarcentred rose on which planetary positions are plotted. Mercury's frequent close approach provides the starting point for an electro-magnetic resonance in the Sun, which is also evident 43 days later when the planet has moved by $180^{\circ}$ - to the opposing resonant position at $225^{\circ}$.

This phenomenon is visible as extensions of magnetised plasma from the solar corona in a four-leaf-clover pattern (which can be replicated by permanent magnets placed near a television screen). It appears that there are four resonant positions $90^{\circ}$ apart; and each is visited in turn by Mercury average 22-day intervals. Clearly, the Sun is in a permanent state of resonance - and its ringing is dramatically enhanced as additional planets pass through one of these four resonant alignments. When viewed from 'above' (from a vantage point above the Sun's North Pole - ie. directly above its axis of rotation) these alignments form a cross on the Sun's equatorial plane.

\section{When will it rain?}

Like all Australian farmers, I had a question uppermost in my mind: When is it going to rain? The conclusions of my 26 years of research and analysis were recently tested on Australia's Gold Coast, where water supply remains a heated issue in times of

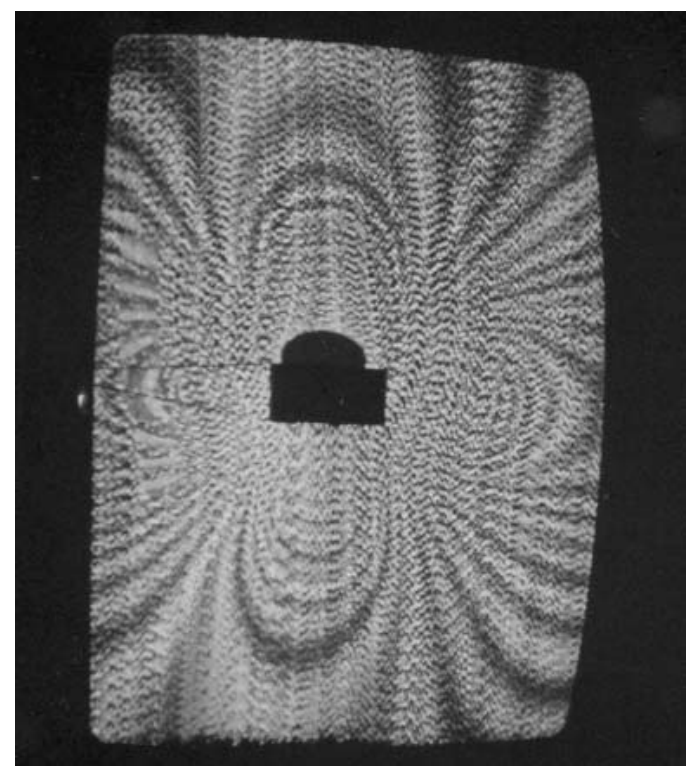

TV screen influenced by permanent magnets. This image serves as the speculative surrogate (viewed from above the Sun's pole) for a four-lobed beam of magnetised plasma radiating "horizontally" onto the ecliptic plane, as maintained by resonant excitation from near-orbiting Mercury. 
current drought. My prediction for future rainfall featured in a local newspaper on 12 July 2002. The article related how weather patterns are a consequence of the factors affecting sunspots; and the parched Gold Coast could expect dam-filling rains, most likely at the end of 2003. I included a caveat - no volcanic eruption should eventuate in the near-Asian region to mask impacts associated with my predicted high sunspot numbers.

In the event, there were no major volcanics; and as predicted; the Hinze Dam catchment received sufficient rain in December 2003 - March 2004 to lift waterstorage levels to $70-80 \%$ of capacity. Was this proof at last of my theories? No, of course not. But I was encouraged; and this paper is the result.

\section{METHODOLOGY}

\section{Landscheidt paper}

On the galactic scale, our solar system is a ball of magnetised plasma, now crossing the Sagittarius-Carina arm of the Milky Way. The solar wind keeps this ball (heliosphere) inflated, and hence affords an inhabited planet a modicum of protection from galactic influences. But inside the heliosphere, there are complex inertial and electro-magnetic relationships which affect the Sun and all the planets. Already in this Journal (Landscheidt 2003), an important element of the planetary connection with Earth and Sun has been elucidated.

That ground-breaking paper helps explain the variability of the solar wind, and hence the degree to which bombarding cosmic rays can enter our atmosphere. The Sun and giant outer planets (moving outward: Jupiter, Saturn, Uranus and Neptune - plus tiny Pluto) orbit the centre-of-mass of the solar system (barycentre). Much of the system's angular momentum is held by these giants, and their collective orbital progress applies a variable torque to the Sun, thus driving its irregular orbit. Rapid rate-of-change of applied torque correlates with increased solar eruptive activity, and a more powerful solar wind.

It has been hypothesised that a stronger solar wind means a better-protected Earth, which means, in turn, reduced penetration of the atmosphere by ionising cosmic rays. Greater influx of electro-magnetic energy also serves to accelerate precipitation of preexisting atmospheric ions. Both influences should reduce cloud-forming nucleation, cloudiness, albedo (reflectivity), and enable an increased quantity of the little-varying solar radiant-energy to reach the surface. The result would be a warmer Earth.

Landscheidt calculated that there will be a decrease in the rate of change of solar torque, and hence the incidence of coronal eruptions, in the decades immediately ahead. Correlation with similar low-torque periods in the palaeoclimatic record, indicates the likely development of cool conditions - such as in the Maunder Minimum of the Little Ice Age - by 2030. People starved then.

The work described above, dealt primarily with momentum effects and their outcome. But there are also other planetary influences on Sun and Earth of a dominantly electro-magnetic nature. While these too involve the outer giants, they also confer a vital role on the small inner planets which orbit the Sun directly (moving outward: Mercury, Venus, Earth and Mars). These effects are described below. 


\section{New concepts}

There are two separate types of planetary conjunction which appear to be particularly influential - based on their observed concordance in timing with known solar/terrestrial events. Correlations are not proof; and unreserved acceptance their message must be deferred until that happy time when understanding catches up with observation.

First is the Angular Separation (AS) of planets, with respect to a circle at the plane on which the planets orbit (ecliptic) and centred on the Sun. When two planets occupy relative angular positions on the orbital plane yielding separations such as $0,30,60$, $72,90,120$ and 180 degrees (divisible into the $360^{\circ}$ whole) resonance is the result.

Second is the Solar Electro-magnetic Resonant Cross (SER-X) where any planet arriving at an azimuth of 75, 165, 255 and 345 degrees (forming the arms of a cross), causes enhanced resonance. SER-X alignments have an impact on sunspot abundance; and also are likely to draw a detectable response from Earth.

The combination of AS and SER-X facilitates the release of energy from the Sun, as evidenced (albeit perhaps, counter-intuitively) by decreasing sunspot prevalence at that precise time. Other contributing factors also relate to planetary orbits. An outer planet with a slow angular velocity (giant Uranus has an orbital period of 84 years, $c f$. Earth's one year) holds a SER-X alignment for longer than does an inner planet. A planet's influence is also likely to be greater when closest to the Sun (perihelion), and longer-lasting when farthest away (aphelion).

By convention, Earth's position relative to the Sun at the 22-23 September Equinox marks the point of zero azimuth on the solar-centred celestial circle, viewed with a Northern Hemisphere perspective. Earth moves (anti-clockwise, as do all planets) to the $75^{\circ}$ SER-X azimuth - corresponding to Mercury's perihelion - at 7-8 December. Unsurprisingly, December is a time of unsettled conditions for Earthlings.

\section{New puzzles}

Stopping there would leave us with a not-simple, but not-too-complicated, message. But in truth, observational evidence suggests a profoundly complex picture. The angular dimension of a SER-X position inflates in accordance with the greater distance of the triggering planet from the Sun. In the case of proximate Mercury (at 0.4 Angstrom Unit), resonant contact is maintained while the planet moves through one degree of its orbital progress. Further out, Jupiter (5.2 AU) appears to maintain solar excitement over $1.5^{\circ}$. However, on reaching distant Pluto (34.4 AU), the single observation as yet available indicates that resonance was enhanced for a period of over four years, as the planet travelled from $5^{\circ}$ before, to $5^{\circ}$ after, the SER-X position of $255^{\circ}$.

But there is another, even more complicating, observational puzzle to be confronted. Sunspots are readily visible from Earth - as is their progress across the face of the Sun. The Sun's rate of rotation varies; and the solar day is about 24 Earth days at its equator, extending to over 30 days at the poles. As one might expect, sunspots are stationary; and their apparent movement is explained by solar rotation. But the highly-variable 'beam' of energetic particles ejected from the four Mercury-dictated SER-X portals is fixed relative to the solar-centred plane on which planetary orbits are described. In direct contradiction to sunspots, in celestial terms they are stationary; but in solar terms they rotate. Clearly, scientific understanding has much catching-up to do. 


\section{Example: the 2001 sunspot peak}

Sunspots provide a readily-observable and convenient proxy for solar eruptive activity, and hence for the intensity of the solar wind. Whitehouse (2005), and many others, carry plots of sunspot observations back to the introduction of telescopes to observatories in the early 1600s. Observations reveal, after a virtual absence from 1645 to 1715 , prominent 9-13 year cyclicity in sunspot abundance, which still continues. A paper in this very volume (Archibald 2006, Figure 1), illustrates past and projected cycles.

Soon and Yaskell (2003) tell us that as early as 1800, William Herschel had noted a correlation between diminished sunspot prevalence and low-crop-yield years in England. Herschel subsequently read at a sceptical Royal Society the paper on his findings, thus elevating from astrology to astronomy the concept of an Earth subject to external influences.

Little Pluto, which has a long period (248 years) and an eccentric orbit, has remained relatively close to its perihelion for the past 30 or so years. Figure 1 illustrates planetary relationships in March 2001. Pluto was in the SER-X position at $255^{\circ}$; and massive Jupiter was $180^{\circ}$ away straight through the Sun - in the SER-X position at $75^{\circ}$. There would have been a powerful enhancement of solar resonance, although with Jupiter's orbital period of 12 years, it could not have been narrowly circumscribed in time.

Why then was there a record sunspot count (352) on the $30^{\text {th }}$ day of March? I speculate that it was because of the additional impetus provided at that time by a short-

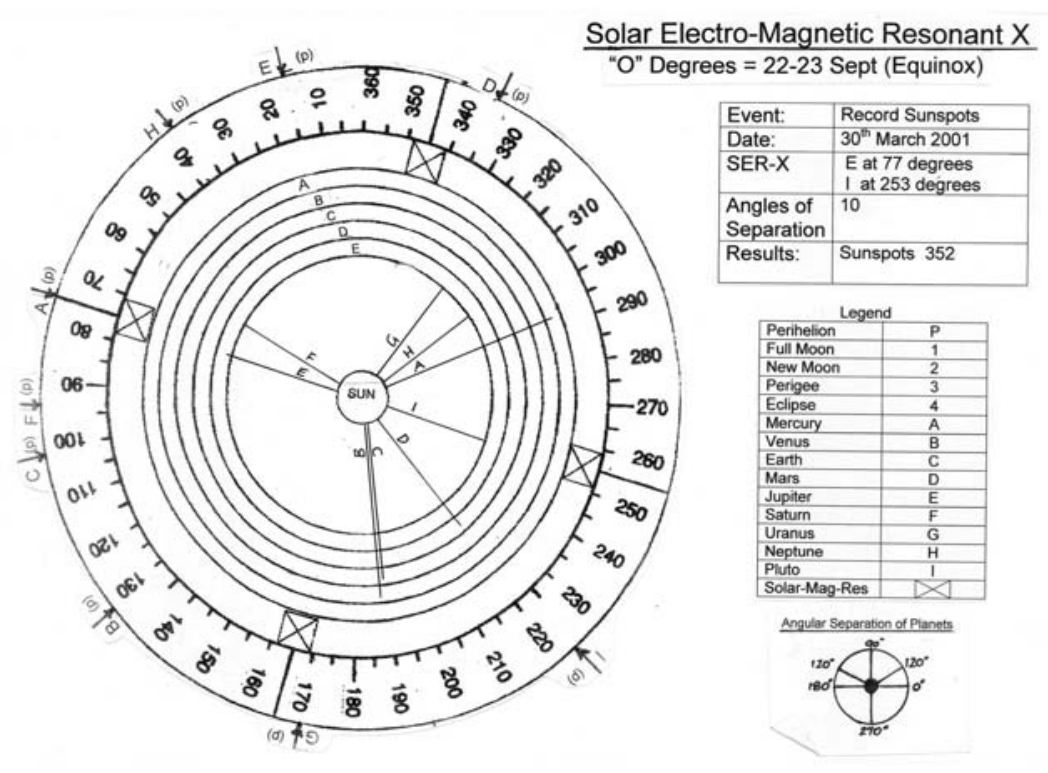

Figure 1: Record sunspot count, 30 March 2001. Jupiter and Pluto were in diametrically-opposed SER-X positions. They and Earth, Venus, Neptune, Mars and

Mercury were also in an unusually-large series of resonant angular separations. 
term assemblage of specific angular separations. As can be seen in Figure 1, Earth and Venus (period $0.6 \mathrm{yr}$ ), both rapidly-orbiting planets, shared an angular position $60^{\circ}$ from Pluto, and $120^{\circ}$ from Neptune. Also, Mars had an AS of $30^{\circ}$ from Pluto and $72^{\circ}$ from rapidly-orbiting Mercury (period $0.24 \mathrm{yr}$ ); and, as already mentioned, Jupiter and Pluto had an AS of $180^{\circ}$. In addition, Pluto was $30^{\circ}$ and Jupiter $60^{\circ}$ past perihelion, and Saturn $30^{\circ}$ before it. (The perihelion locations of all planets are shown on the circumference of the celestial chart in Figure 1.)

Is this a valid explanation, or an opportunistic reliance on coincidence? I say it would be more of a coincidence for such a remarkable assemblage of resonant angularities to have had no detectable physical outcome.

\section{PLANETARY CONTEXT FOR ASIAN TSUNAMI}

On 24 December 2004, an earthquake of 8.1 R (Richter Scale) magnitude was recorded beneath the Southern Ocean some $800 \mathrm{~km}$ SE of Hobart. Happily, there was no damage. However, a following $(9.3 \mathrm{R})$ 'quake under the Indian Ocean on 26 December led to the devastating Asian Tsunami. Those who attributed the triggering of this seismic sea-wave to human decadence, or indeed any terrestrial cause, were probably mistaken. Figure 2 provides a more-plausible explanation.

The scene was set by a series of contributing factors: On the $24^{\text {th }}$, Saturn (period 29 yrs) was only $10^{\circ}$ past its $93^{\circ}$ perihelion; and Earth was at $93^{\circ}$ - only 10 days before its

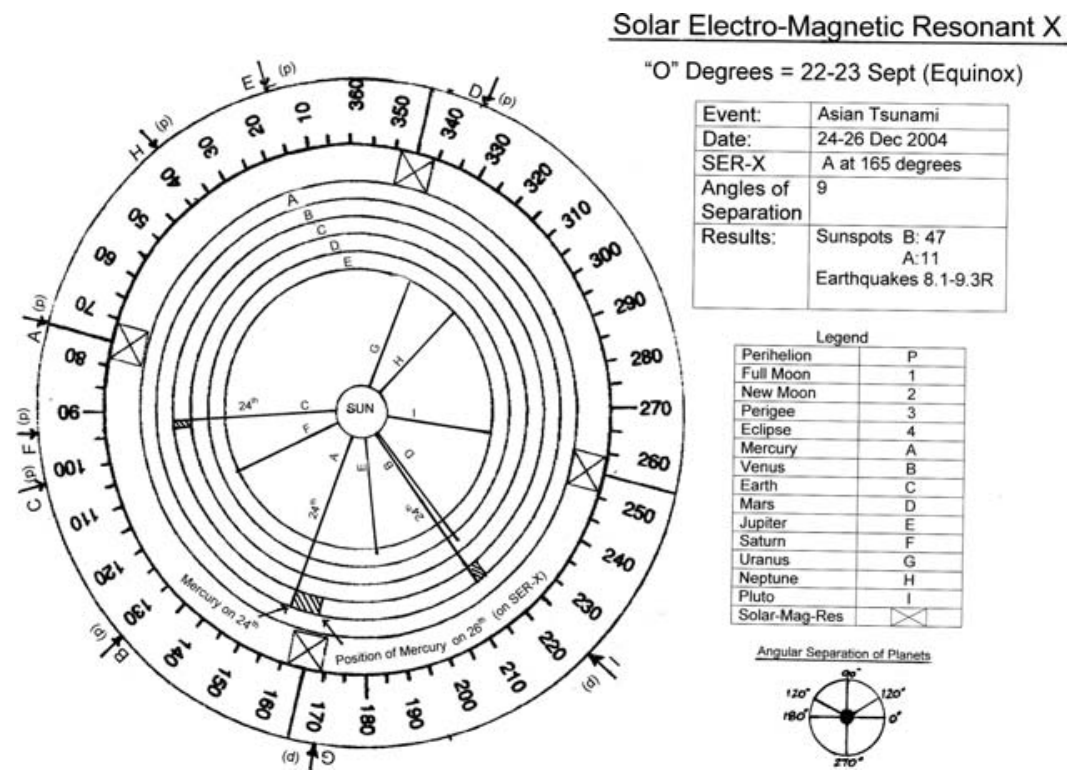

Figure 2: Asian Tsunami, 26 December 2004. Angular separations of $60^{\circ}$ between Earth and Mercury, and between Mercury and Venus/Mars, were present at the time of the first earthquake on the $24^{\text {th }}$; an AS of $120^{\circ}$ also existed between the latter pair and Uranus. Rapidly-orbiting Mercury had reached SER-X of $165^{\circ}$ by the time of the second 'quake on the $26^{\text {th' }}$. 
own perihelion. Pluto was still in a $4-\mathrm{yr} 74^{\circ}$ angular relationship with Uranus (period 84 yrs) - which was itself approaching aphelion. A notable AS also existed at that time between Earth-Mercury-Venus/Mars with their $60^{\circ}$ separations being the identical harmonic of the orbital circumference. At this time, the first 'quake was triggered.

Two days later, fast orbiting Mercury $\left(90^{\circ}\right.$ past perihelion) attained the $165^{\circ} \mathrm{SER}$ $\mathrm{X}$ position. This was the very day of the second 'quake - when the lithosphere would still have been ringing with the shock of the first. Coincidence? We shall never know. But there is disinterested corroboration - from none other than the Sun. A remarkable change in sunspot abundance was observed over the crucial period. It went from 47 on the $23^{\text {rd }}$, down to only 11 on the $27^{\text {th}}$, and by the $31^{\text {st }}$, it had rebounded to 60 . Wellunderstood or not, extra-terrestrial influences were in evidence at that fateful time.

\section{ABRUPT END TO 1997/8 EL NIÑO}

As described elsewhere in this volume (McLean 2006), the El Niño/La Niña (ENSO) alternation is a crucial determinant of rainfall in water-short Australia. The seasonal nature of the variable upwelling which drives Pacific sea-surface temperature (SST) is well illustrated by Guilderson and Schrag (1998, Figure 2); and a detailed analysis of the great 1997/8 El Niño is also presented herein (Kininmonth 2006), in which the wider climatic implications of such events are explained.

The growth, maturity, and collapse of this prominent climatic event is enshrined in the SST record from a buoy on the equator at $125^{\circ} \mathrm{W}$ longitude (McPhaden 2002 , Fig 1-15). Build-up began in normal seasonal fashion in January 1997, with SST rising from an atypically cool $23^{\circ} \mathrm{C}$ to the warm-season norm of $27^{\circ} \mathrm{C}$ by March. In a 'neutral' ENSO year, SST would have risen no further; and then, renewed upwelling in the eastern equatorial Pacific would have caused cooling to $24^{\circ} \mathrm{C}$ by August. However, in this case, SST continued to rise (albeit at a lower rate) to attain a needlepeak of almost $30^{\circ} \mathrm{C}$ in early May 1998 .

A sudden reversal then took place. By the end of the month, SST was back to $23^{\circ} \mathrm{C}$ - thus over-shooting the seasonal cooling expected several months later in a time of neutral regime. At this equatorial site, SST fell by a climatically-significant $6+{ }^{\circ} \mathrm{C}$ in less than a month! In November, full La Niña conditions arrived, after a second (lesser) cooling step took SST to below $21^{\circ} \mathrm{C}$.

Figure 3 shows planetary conjunctions on 12 May 1998. The El Niño appears to have been in part an outcome of the $60^{\circ}$ AS between Pluto and Uranus; but their separation had widened to $64^{\circ}$, suggesting that this resonance had now been lost. But, many new influences emerged. Earth enjoyed close to a $72^{\circ}$ AS from Mercury, Venus and Neptune; and, equally significant, Jupiter had achieved SER-X of $345^{\circ}$. Lastly, all planets were on the same side of the Sun, travelling in the same direction with Earth trailing the field. A reduced bow-shock for Earth's protective magnetosphere would have been a possible outcome.

The abrupt decay of the giant 97/8 El Niño in May 1998, and its subsequent replacement by a fully-developed La Niña in the following November, may have been the direct result of sharp changes in planetary alignments. Implausible? Maybe so. But any exclusively-Earth-bound explanation so far proffered up for this remarkable reversal, is even more implausible. 


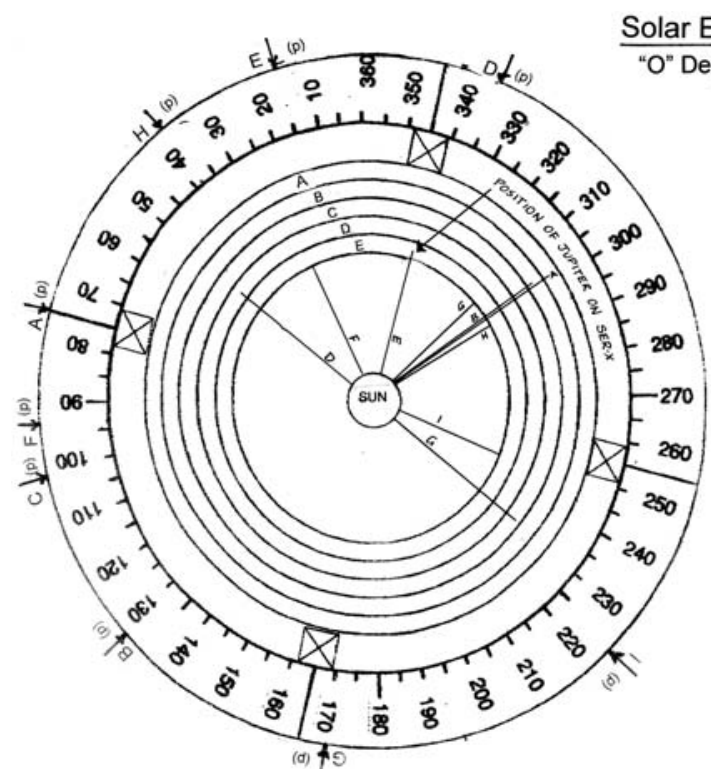

Solar Electro-Magnetic Resonant X " Degrees $=22-23$ Sept $($ Equinox

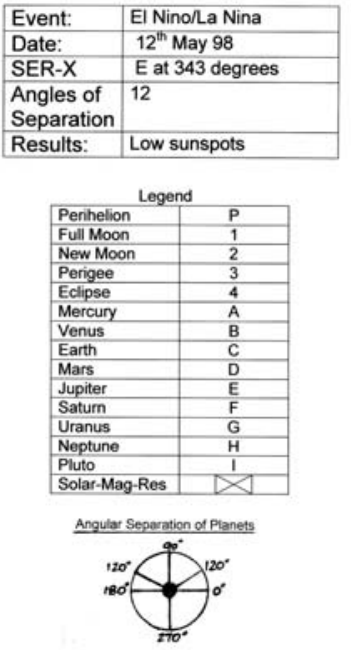

Figure 3: Abrupt El Niño/La Niña reversal, 12 May 1998. All planets were on the same side of the Sun, with Earth last in line; and there was a $72^{\circ}$ angular separation between Earth and Neptune/Venus/Mercury. Jupiter was on SER-X at $345^{\circ}$.

\section{MAUNDER MINIMUM WAS EXTRA-TERRESTRIALLY DRIVEN}

The latter millennia of the present (Holocene) Interglacial have exhibited a cooling trend, overprinted by a ca. 1500-year warmer-cooler cyclicity. In the temperate Northern Hemisphere its most-recent manifestations were the climatic/historical Roman Empire Warm Period (Romans in England), Dark Ages (Germanics in Italy), Mediaeval Warm Period (Vikings in Greenland) and Little Ice Age (LIA).

The LIA was prominently twin-troughed (Wang et al 2005); and its Spörer and Maunder cold periods were times of great hardship in Europe. The Maunder Minimum, for which we have the better records, took the form of an intermittent series of very cold winters. The subsequent 300 -year trend to present-day warmth, has been interrupted by three progressively less-and-less severe cold intervals at 1800-20 (Dalton Minimum), 1880-1910, and 1950-75. There is little doubt that the timing of LIA minima correlates with variation in sunspot abundance and cosmic ray flux (Usoskin et al 2005). But what is their underlying driver? Why, for instance, were there almost no sunspots between 1645 and 1715 ?

I now introduce the culprit. Pluto, with its huge orbit, was in its aphelion; and it simultaneously held a SER-X position (Figure 4). Neptune was also in aphelion; and it also held a SER-X position. With Uranus heading towards its aphelion as well, the Sun appeared to lose contact with the outer planets. By 1715, however, conjunctions were dramatically different. Two of the outer planets were in perihelion (Uranus, Neptune), while Pluto had travelled 90 degrees to hold the next SER-X position (the only time a simultaneous occurrence of this type has been noted). These outer planets 


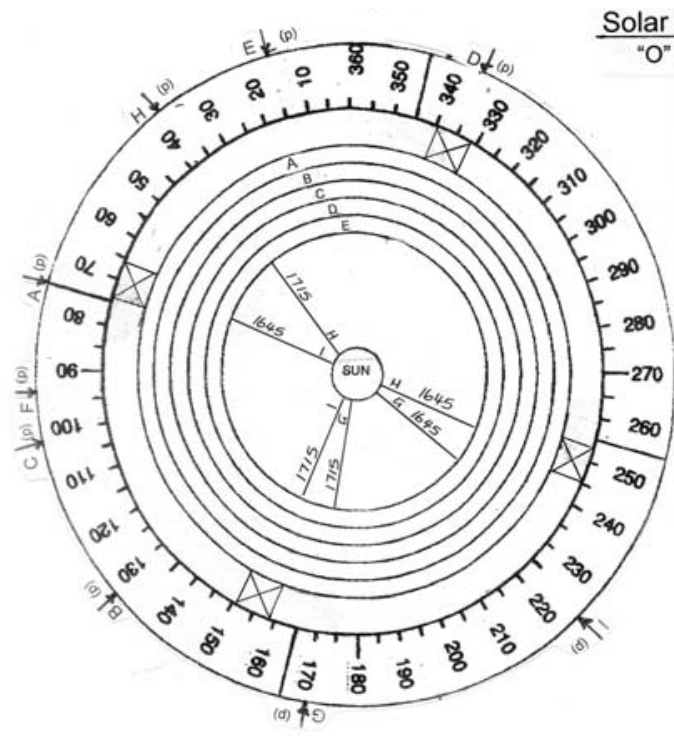

Solar Electro-Magnetic Resonant X

"O" Degrees = 22-23 Sept (Equinox)

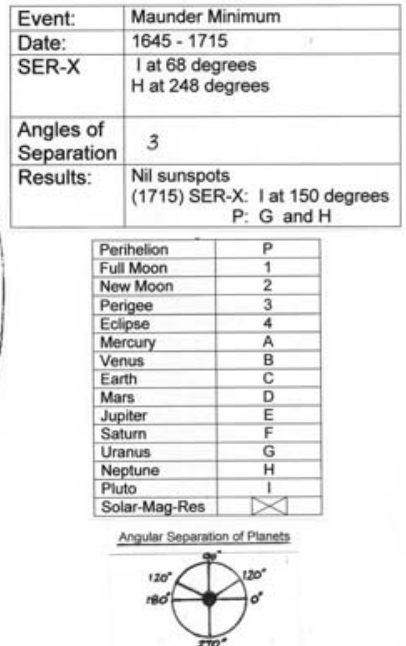

Figure 4: Outer planets during the Maunder Minimum. In 1645, Pluto and Neptune were $180^{\circ}$ apart, both in SER-X positions and on aphelion - with Uranus approaching aphelion. By 1715, Pluto had moved to another SER-X position, and both Uranus and Neptune were now on perihelion.

were back! The Sun was re-energised; and the 300-year warming trend we still enjoy had begun.

My analysis above does no violence to Landscheidt's currency. He dealt in the rateof-change of torque applied to the Sun by the collective orbital motions of the outer planets. An individual planet's influence is greatest when its angular velocity is greatest - at or near perihelion; and the combined influence of two planets is greatest as one passes the other. At its simplest, planets in aphelion 'switched off' the Sun in 1645, and planets at perihelion switched it on again in 1715. The Maunder Minimum "quiet Sun" can be thus explained; and there is little reason to doubt that this notable climatic event occurred, and that it was extra-terrestrially driven.

\section{FURTHER POSITIVE CORRELATIONS}

Computer analysis identifying significant angular relationships, in conjunction with my theories of planet/SER-X alignments, enables identification of solar-induced terrestrial disasters. When multiple planets connect in resonance-positive AS around the Sun, and with at least one planet holding a SER-X position, the result can be chaos on Earth. The following notable events correlate with SER-X alignments:

- Volcanic eruptions - Tambora 1815; Krakatau 1883; Pelée 1902; St. Helens 1980; Pinatubo 1991.

- $\quad$ Earthquakes - San Francisco 1906 and 1989; Kobe 1995; Asian Tsunami 2004. 
- Long-distance power line overloads - Quebec 11-17 March 1989; USA 10-12 January 1997 and 14-15 August 2003.

\section{WARNING - UNUSUAL CONJUNCTIONS IN JUNE 2006}

As shown in Figure 5, there will be a remarkable four planets holding SER-X positions on 6-7 June 2006. I am not familiar with a previous such event. Mercury will be at SER-X directly opposite Venus/Uranus; and Earth will hold SER-X at right angles.

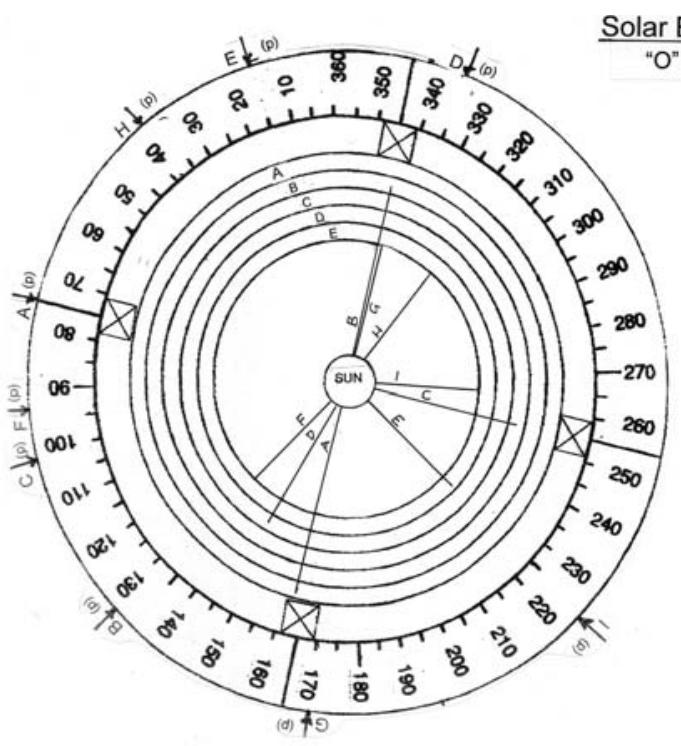

$\frac{\text { Solar Electro-Magnetic Resonant X }}{\text { "O" Degrees }=22-23 \text { Sept (Equinox) }}$

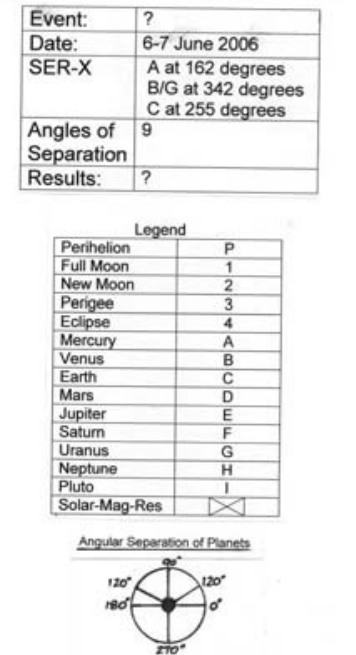

Figure 5: Exceptional planetary conjunctions on 6-7 June 2006. No less than four SER-X alignments will happen simultaneously - Mercury at $165^{\circ}$, Earth at $255^{\circ}$, and both Venus and Uranus at $345^{\circ}$. In addition, there will be several angular separations: Venus-Uranus $0^{\circ}$, Saturn-Mercury $30^{\circ}$, Mercury-Jupiter $60^{\circ}$, Mercury-Earth $90^{\circ}$, Saturn-Jupiter and Jupiter-Neptune ca. $90^{\circ}$, Mars-Pluto ca. $120^{\circ}$, and Mercury-Venus/Uranus $180^{\circ}$.

Furthermore, there will be a plethora of resonant angles in force. For instance, fastorbiting Mercury will have an AS, not only of $180^{\circ} \mathrm{C}$ with Venus and Uranus, but of $30^{\circ}$ with Saturn, $60^{\circ}$ with Jupiter, and $90^{\circ}$ with Earth. Other less-precise conjunctions include $94^{\circ}$ Saturn-Jupiter, $118^{\circ}$ Mars-Pluto, and $92^{\circ}$ Jupiter-Neptune.

Because of the directional nature of solar energetic outbursts, particularly coronal mass ejections, we cannot be sure that Earth will be involved. However, another earthquake is among the possibilities. But what value is an alert which gives timing without location? Recent advances may help. Earthquake prediction, based on atmospheric factors, is under development. It appears that satellite-aided detection of cloud lineaments along known fault lines may offer one or two day's notice. Lives could be saved. 


\section{CONCLUSIONS}

- Energy distribution in the solar system varies because of planetary dynamics.

- Any analysis of cause and effect for observed natural events on Earth is incomplete, unless and until it includes recognition of appropriately-timed extraterrestrial forcing.

- Inevitably, projection of human wellbeing across the century ahead is bound to be false and misleading, should it exclude consideration of the potential for short and longer-term planetary influences upon terrestrial fortunes.

\section{REFERENCES}

Archibald, David C. 2006, "Solar Cycles 24 and 25 and predicted climate response", Energy \& Environment v. 17 no. 1 (in press).

Guilderson, J. T. and Daniel P. Schrag 1998, "Abrupt shift in subsurface temperatures in the tropical Pacific associated with changes in El Niño", Science v. 281 pp. 240-3.

Kininmonth, William 2006, "The El Niño phenomenon in the context of climate change", Energy \& Environment v. 17 no. 1 (in press).

Landscheidt, Theodor 2003, "New Little Ice Age instead of global warming?", Energy \& Environment v. 14 no. 2, 3 pp. 327-50.

McLean, John D. 2006, "A critical review of Australian climate reports", Energy \& Environment v. 17 no. 1 (in press).

McPhaden, Michael J. 2002, "El Niño, La Niña, and the climate swings of 1997-98”, pp. 25-30 in Michael H Glantz (Ed) "La Niña and its impacts: facts and speculation", United Nations University Press $271 \mathrm{p}$.

Soon, Willie Wei-Hock and Steven H. Yaskell 2003, "The Maunder Minimum and the variable Sun-Earth connection”, World Scientific 278 p.

Usoskin et al 2005, "Solar activity, cosmic rays, and Earth's temperature: A millennial-scale comparison", J. Geophys. Res v. 110, A10102, doi:10.1029/2004JA010946, 11 p.

Wang, Yongjin et al 2005, "The Holocene Asian Monsoon: links to solar changes and North Atlantic climate", Science v. 308 pp. 854-7.

Whitehouse, David 2005, “The Sun: a biography”, Wiley 334 p. 
